\title{
UBICACIÓN LABORAL DE LOS EGRESADOS DE LA FACULTAD DE ENFERMERÍA
}

María C. Melo B., Liliana P. Moreno H., Luz.A. Piñeros S., Consuelo M. Rodríguez B.*

\section{Resumen}

La finalidad de esta investigación es identificar la ubicación laboral de los egresados de la Facultad de Enfermería de la Fundacion Universitaria de Ciencias de la Salud y su desempeño en los campos de formación establecidos en el perfil profesional. Para desarrollar este trabajo se recopilaron datos de los archivos de la facultad, entre julio de 1994 y junio de 1999, con un total de 363 egresados, de los cuales se ubicaron 166 y respondieron 111. Los egresados (73\%) en los años de 1996-1998 con límites de edad entre 18-41 años (promedio de 26 años), están ubicados en Santafé de Bogotá, con un nivel socioeconómico medio, en mayor porcentaje; en los campos asistencial, de docencia y gerencia se desempeñan $92 \%$. El nivel académico de los egresados se ajusta a la realidad del país y la formación dada por la facultad es adecuada.

\section{Introducción}

La finalidad de esta investigacion es establecer la ubicación laboral de los egresados de la Facultad de Enfermería de la Fundacion Universitaria de Ciencias de la Salud; determinando si se desempeñan en los campos de formación establecidos en el perfil profesional como son: asistencial (clínico-comunitario), docencia, gerencia, investigación y ejercicio independiente, los cuales son ofrecidos por la facultad que tiene como propósito capacitarlos de una forma activa, responsable y honesta, en busca de la formación del estudiante dentro de un proceso dinámico y evolutivo, tomándolo como un ser biosicosocial, para un desempeño exitoso en los campos de trabajo en donde demuestre permanentemente una actitud de liderazgo.

Esta investigación permite reforzar y ampliar la información acerca del desempeño de los egresados ya que es indispensable establecer si es necesario someter a consideración los programas aplicados actualmente y presentar sugerencias para la participación de los mismos desde el campo profesional,

\footnotetext{
Facultad de Enfermería; Fundación Universitaria de Ciencias de la Salud, Hospital de San José, Bogotá D.C.
}

siendo útil para la institucion ya que permite dar a conocer si los profesionales de enfermería se desempeñan en todos los campos de formación y así poder enriquecer el curriculum o hacer los correctivos necesarios con respecto a los resultados obtenidos. Es novedosa ya que la institucion no cuenta con este tipo de información que le permita tener un conocimiento acerca de sus egresados, con el fin de enriquecer el curriculum y obtener información para la toma de decisiones, teniendo como objetivo general determinar la ubicación laboral de los egresados de la Facultad de Enfermería de la Fundacion Universitaria de Ciencias de la Salud según los campos de formación establecidos en el perfil profesional que ofrece la facultad, en el período julio 1994-junio 1999.

La base teórica de esta investigacion fue fundamentada en: reseña histórica de la Fundacion Universitaria de Ciencias de la Salud y elementos curriculares, que comprenden la filosofía y los objetivos. La filosofía considera indispensable desarrollar en el egresado la capacidad de análisis de la realidad, iniciativa, creatividad y habilidad para que actúe con alto sentido ético y humanístico, al reconocer al docente como orientador y dinamizador del proceso 
enseñanza-aprendizaje, consciente de la importancia del desarrollo profesional, junto con el avance científico y tecnológico en enfermería.

Por su parte, para los objetivos, el profesional de enfermería está capacitado para: brindar cuidado integral al individuo, la familia y la comunidad en las diferentes situaciones clínicas, a través de las acciones de enfermería, desarrolladas para satisfacer las necesidades del paciente; proyectar una actitud de liderazgo en la coordinación con el equipo interdisciplinario y en la supervisión de acciones asignadas al personal a su cargo; participar y fomentar la investigación según las necesidades identificadas en su área de desempeño.

Los campos de formación dados por la Facultad, comprenden:

Asistencial (clínico-comunitaria): comunicación directa con el entorno y el equipo de salud y la planeación de acciones de enfermería para recuperar la salud y rehabilitar al individuo.

Docencia: aplicar el proceso enseñanza-aprendizaje en la formación del profesional de enfermería y dar educación en salud al individuo, la familia y la comunidad.

Gerencia: buscar un diagnóstico de necesidades en materia de recursos humanos, financieros y materiales.

Investigación: de tipo interdisciplinario de acuerdo a las necesidades identificadas en su área de desempeño, elaboración, presentación de proyectos y desarrollo de programas de educación.

Ejercicio independiente: aspecto teórico-conceptual consignado en el perfil profesional que la facultad considera como formación integral e indispensable para su adecuado desempeño.

\section{Metodología}

Esta investigación corresponde al paradigma cuantitativo ya que implica la recolección de información numérica y el análisis por medio de procedimientos

estadísticos; es descriptivo porque determina la situación de las variables y es transversal pues la información se obtuvo en un momento único; se realizó en Santafé de Bogotá, tomando como base los archivos de la Facultad de Enfermería para determinar la ubicación geográfica de los egresados. La población es de 363 egresados de enfermería desde julio de 1994 a junio de 1999; se obtuvo una muestra de 166 egresados a quienes se les envió el formulario y respondieron 111.

Al realizar el análisis estadístico se obtiene una muestra que es representativa para el estudio de tipo descriptivo, con un margen de error de $5 \%$ y que arroja los siguientes datos: de los encuestados: $73 \%$ han egresado en los años 1996-1998, discriminados así: 25\% en el año de 1996, 20\% en 1997 y 28\% en 1998. En los años de 1996-1997 el número de egresados en el segundo período académico de cada año corresponde a 15,3\% del total de encuestados, es decir $30,6 \%$ para ambos años, con 17 egresados en cada semestre. En 1998 el mayor número de egresados por período académico se presentó en el primer semestre con 22 egresados (20\%), igualando el total de egresados del año inmediatamente anterior (Tabla 1).

\section{Composición de egresados por año vs. período académico}

\begin{tabular}{|c|c|c|c|c|}
\hline \multirow[b]{2}{*}{ AÑO } & \multirow[b]{2}{*}{ ler sem. } & \multicolumn{3}{|c|}{ Período académico } \\
\hline & & 2do sem. & Total & Porcentaje \\
\hline 1994 & 0 & 8 & 8 & 7 \\
\hline 1995 & 4 & 12 & 16 & 14 \\
\hline 1996 & 11 & 17 & 28 & 25 \\
\hline 1997 & 5 & 17 & 22 & 20 \\
\hline 1998 & 22 & 9 & 31 & 28 \\
\hline 1999 & 6 & 0 & 6 & 6 \\
\hline TOTAL & 48 & 63 & 111 & 100 \\
\hline
\end{tabular}

Tabla 1

Los encuestados se encuentran en un rango de edad comprendido entre los 18 y los 41 años de edad, 89\% tiene entre 18 y 33 años y $11 \%$ entre 34 y 41 años de edad. La edad promedio es de 26 años y el rango de edad donde mayor número de egresados se tiene es el de 18 a 25 años, con $47 \%$. 
De los egresados 107 están en el país, con sede en Santafé de Bogotá; de ellos, 95\% se ubican en el nivel socioeconómico medio, con $3 \%$ en el nivel alto y $2 \%$ en el nivel bajo (Tabla 2 ).

\section{Nivel socioeconómico}

$\begin{array}{lcc}\text { Nivel } & \text { No. egresados } & \text { Porcentaje } \\ \text { Alto } & 3 & 3 \\ \text { Medio } & 106 & 95 \\ \text { Bajo } & 2 & 2 \\ \text { Total } & 111 & 100\end{array}$

Tabla 2

Para quienes han realizado especialización, las áreas de preferencia son Unidad de Cuidado Intensivo (35\%) y gerencia (53\%) (Figura 1).

\section{Especialización de los egresados}

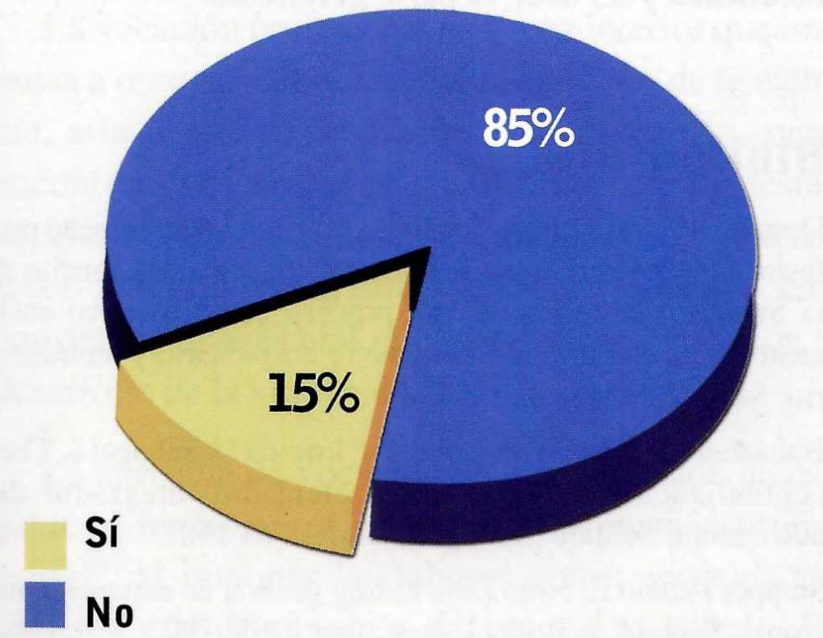

Figura 1. Entre quienes han realizado especialización (15\% de los egresados), las áreas de preferencia son Unidad de Cuidado Intensivo (U.C.I.) y gerencia.

Tan sólo 5\% de los encuestados no ejercen la profesión de enfermería y otro 95\% la están ejerciendo en el momento de la encuesta. De 105 egresados que ejercen la profesión, $50 \%$ tienen menos de 30 meses de ejercerla y $50 \%$ superan los 30 meses de ejercicio para un promedio de $30,6 \%$ meses de ejercicio profesional (Figura 2).

\section{Tiempo de ejercicio profesional}

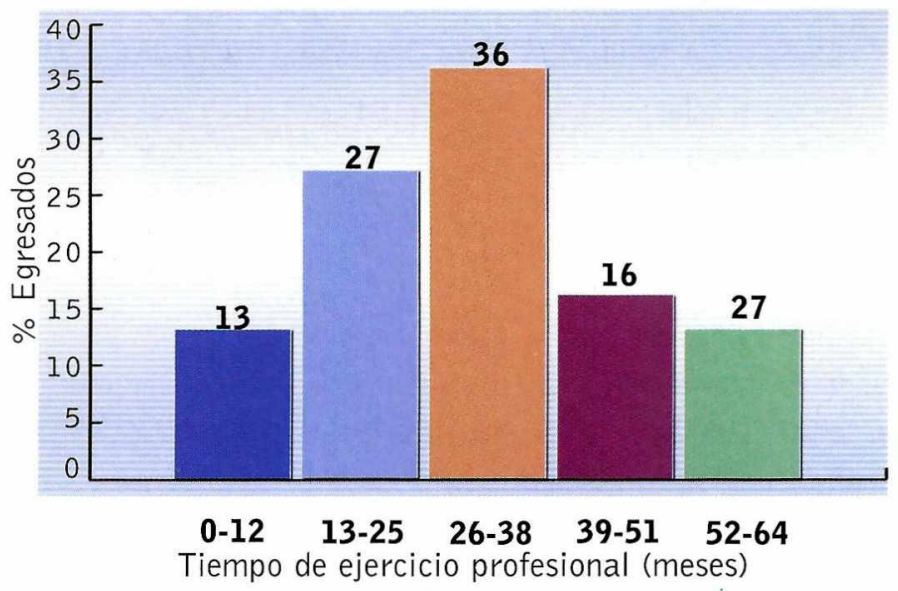

Figura 2. Del total de los egresados encuestados, 105 ejercen actualmente la profesión, de los cuales la mitad tiene menos de 30 meses de ejercicio.

Los datos mostraron que $92 \%$ de los encuestados se han, o se desempeñan, en los 3 primeros campos de formación como son: asistencia (clinico-comunitaria), docencia y gerencia, en sus diferentes combinaciones. Al predominar el ejercicio profesional y ocupar cargos en el ámbito asistencial y docente, $8 \%$ combinan los niveles asistencial, docente, gerencial, investigativo y el ejercicio independiente, lo cual indica el predominio del ejercicio profesional en los 3 primeros niveles de egresados de la Facultad de Enfermería de la Fundación Universitaria de Ciencias de la Salud (Tabla 3).

Las instituciones y servicios en los cuales se han desempeñado los egresados son: en el área asistencial en hospitales, clínicas y otras instituciones, fundaciones y CAMIs; los servicios corresponden a las áreas de UCI, adultos y neonatos, ortopedia, medicina interna, unidad renal, urgencias, pediatría, cirugía, obstetricia, consulta externa y gerencia hospitalaria. Las personas que se desempeñan en docencia se encuentran en instituciones y universidades.

La formación académica es suficiente para el desempeño profesional, según $72 \%$ de los egresados, porque las prácticas se realizan en escenarios apropiados en donde se confronta la academia con la realidad. Consideran también que el nivel académico es bueno por la calidad e idoneidad de sus docentes. Sin embar- 


\section{Desempeño según campos de formación}

\section{Campos de formación}

\section{No. egresados}

Porcentaje
1. Asistencial

2. Docencia

54

30

7

4. Asistencial y gerencia

5. Asistencial e

independiente

6. Asistencial, docencia

y gerencia

7. Asistencial, docencia

e independiente

8.Asistencial, docencia

e investigación

9. Asistencial, docencia, gerencia e investigación

10. Asistencial, docencia, gerencia e independiente

11. Asistencial, docencia, investigación e independiente

Total

108
4

50

4

27

6

1

5

1

1

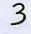

1

1

1

100
Tabla 3

go, 74\% mencionaron que debe hacerse énfasis para ampliar los conocimientos académicos y prácticos en las áreas de quemados, salud mental, área administrativa o de gerencia.

En la formación de los egresados, los recursos necesarios para las actividades son siempre óptimos; sin embargo, la realidad en el desempeño es muy diferente, por lo cual el egresado debe adaptarse al medio; $64 \%$ resaltaron la formación integral del estudiante de la Fundación Universitaria de Ciencias de la Salud ya que, además de los conocimientos específicos en las diferentes áreas, se suma la experiencia de los docentes. Hubo $14 \%$ que se abstuvieron de responder a este punto, por cuanto consideraron no tener los elementos de juicio en el momento de practicarse la encuesta .

\section{Conclusiones}

En la actualidad, 95\% de los egresados de la Facultad de Enfermería, de la Fundación Universitaria de Ciencias de la Salud se encuentran trabajando en los cargos dentro de los campos de formación establecidos por el perfil profesional y están ubicados en Santafé de Bogotá.

De los encuestados, $90 \%$ se desempeñan en los campos asistencial (clínico-comunitario), docencia y gerencia, lo que indica con ésto su nivel de preferencia y de preparación profesional para desempeñarse en las actividades y cargos que demandan las instituciones del sector salud.

El desempeño de los egresados corresponde a la formación y filosofía de la facultad y la formación académica corresponde al desempeño profesional y realidad del país. Llama la atención que un mínimo de egresados practica el ejercicio independiente y del total de los egresados, $47 \%$ han realizado postgrados en clínica y $53 \%$ en la parte gerencial.

\section{Bibliografía}

Domínguez M, Gutiérrez M, Rojas C, Vega J. Desempeño profesional de los egresados de la Fundacion Escuela Ciencias de la Salud en la aplicación del proceso administrativo en la atención de enfermería en el ámbito hospitalario y ambulatorio, Santafé de Bogota 1994.

Buenahora L, Garzón M, Gómez A, Lozada D, Sabogal L. Conceptualización de salud como elemento integrador del curriculum, Santafé de Bogotá julio 15 de 1987.

Samper Pizano E, Niño Díaz L. Ley general de educación nacional, Santafé de Bogotá, diciembre 23 de 1997.

Ley 60 de 1993.

Ley 100 de Diciembre 23 de 1993.

Ley 10 de Enero de 1990.

Ley 266 de 1996.

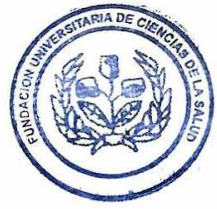

\section{BDLIOTECA}

